\title{
Self and Interdependence
}

First a note on a key term in our book's title. People sometimes misread or misinterpret the word "fractal," thinking it is a fancy way to say "fractured." But the meanings are quite the opposite. Fractal refers to profound continuity and connectedness that in mathematical formulations may propagate infinitely. In this book you will read about numerous examples of fractal organization in the systems of nature and, in human society, discover the powerful potential of the kind of person we call a fractal self. Please read on.

The story that unfolds on these pages tells how complex structure and function, and ultimately life itself, arose by virtue of the astonishing power of cooperation between self and other. Along the way, as our readers will discover, cooperation has proved a catalytic force amid some perhaps surprising conditions of universal being such as turbulence and fractal geometry. We will first explore how those simple physical states have fostered mutualism and emergence in nature, and subsequent chapters will follow this thread deep into the evolution and ethos of human nature.

Some of these phenomena and forces sound like esoteric philosophical abstractions, with vaguely mathematical and scientific overtones. We define them for our readers in accessible terms and trace their influence through evolutionary time to the present. On this journey, cooperation will loom ever larger and more clearly as a cardinal guiding principle of evolution in its physical, chemical, biological, and social manifestations. Today, science is rediscovering these concepts and integrating them with Darwinian theory. We also describe a new framework of evolutionary thought emerging from philosophy and embracing, in its formative roots, a number of ancient traditions, especially those of East Asia. Integrating this new science and rediscovered philosophy, we arrive at surprising prescriptions of how the evolutionary sense of cooperation may optimally shape our lives and open up a hopeful pathway to the future.

Both cooperation and competition were in Darwin's mind, but he and his followers greatly emphasized competition in shaping his great theory of evolution by natural selection. We have focused on new discoveries and ancient wisdom regarding a universal process in which hard edges vanish and disparate structures and functions meld together seamlessly in myriad ways across a range of scale from the atomic to the cosmic. In our book, we have gathered a wide spectrum of thought that has just now begun to pass through the powerful lens of a new scientific revolution focused on the self-organizing universe. This approach is illuminating the integrated properties and behaviors of the systems of nature, 
a way of knowing that philosophers and scientists call holism, which probably traces as far back as the Stone Age. Holism was adopted and developed by the ancient sages of East Asia and the Greek nature philosophers. Holistic thinking opens a window on the universe that is complementary to the perhaps later tradition of reductionism-the prevailing scientific convention that seeks to explain the whole by studying its parts. In the pages that follow, this story opens with a primer on the strangely pregnant patterns and creative powers hidden in the seamless contours of fractal geometry, strange attractors, and the edge of chaos. We then look back in time to our cosmic origins as illuminated by modern science and also as envisioned in strikingly holistic creation myths of various ancient peoples. From these beginnings we proceed on interlaced pathways, scientific and humanistic, toward new perspectives on evolution that, we believe, offer glimpses of a revolutionary synthesis of nature and human nature.

It is our view that the recent formulators of chaos and complexity theories have swept up and crystallized in computational and graphic terms a long and venerable, but chronically suppressed, way of thinking about the origin of the physical universe and its evolutionary patterns, leading to the emergence of life and human society. In this book, we traverse naturalistic philosophies and religions: pre-Socratic philosophy, Confucianism, Daoism, Buddhism, and the mythology of Hawaiian and other premodern cultures. Ultimately, we find a compelling convergence with modern holistic scientific theory-a synthesis that points not only to the evolution of mind-from-nature, but also to a realization that this emergent phenomenon has reached a seminal threshold with the evolution of human beings. In our self-organizing universe, for the first time, conscious creation has appeared. Constructive and destructive behavior in a very small part of the universe is only now backed by purpose. This changes everything.

Perhaps the most striking and frequent marker on this book's less-traveled path to an understanding of evolution and our place in it is emergence. This holistic state of being delights many philosophers and scientists. Its existence was first predicted in formal terms by mathematicians. Emergence is a nonDarwinian phenomenon that results in unpredictable and often powerful new properties or behaviors of systems that develop from interconnecting units such as atoms, polymers, cells, organisms, ecosystems, economies, societies, and so forth. Cooperation in nature is often a trigger for emergence. Around the turn of the twenty-first century, cooperation began to be recognized for its enormous role in shaping biotic evolution. Under the Darwinian microscope, cooperation occurs at random and is then selected for its performance in a competitive milieu. Complexity theory hints at a larger idea. Cooperation, indeed, has long functioned as a front-loader for natural selection, but it is also an expected phenomenon in the universe-a prime attractor that has sometimes catalyzed profound emergent events. Cooperation and competition may frequently oscillate, as do yin and yang. We suggest that virtually throughout evolution, cooperation has held a tiny edge over competition, an advantage probably impossible to measure accurately but one that may represent what we envision as a cooperative constant operating in the universe at large. 
Out of the evolutionary processes leading to complexity in nature, human beings appear to have reached the potential of achieving a seminal state of being in the world-a state we call the fractal self:" Like many other emergent phenomena, a fractal self develops and thrives in conditions that are mildly chaotic: a realm of turbulence with hidden patterns and creative potential. The principle of cooperation is closely aligned with a fractal self, that is, any person drawn to some walk of life, vocation, or avocation who begins to realize a seamless participatory ethos, as a "natural" or an "adept" with growing sensitivity, adaptation, understanding, and expertise. And such a self tends to develop a capacity to foster creative complexity that may lead to positive emergence.

This capacity, however, must be nurtured to overcome self- and societydestructive tendencies if humans are to play a catalytic role in the process of universal evolution. We currently stand at a juncture where no being has stood before. Our world of complex economic, political, and ecological processes is inexorably interwoven as never before imagined. The arrival on this scene of the fractal self points to ways to affect evolution in a positive, hopeful manner. Developing sensitivity vis-à-vis the world, such a self has the potential for shaping a future that holds a burgeoning "ecology of hope." Hence, we discover the fractal self that seeks inclusive participation in nature, as a form of saving grace for our planet and ourselves. This holistic tendency then becomes not only a philosophical and scientific issue, but also a spiritual standpoint that projects itself to a benign future.

In raising ideas at the interface of philosophy and science, our language is highly metaphorical. Metaphor has traditionally been more at home in philosophical discourse than in scientific reporting. Yet metaphor has found wide and effective use among scientists. We believe this literary device is especially useful in the early stages of grappling with concepts the logical structures and operating principles of which are partially veiled. Like electrons scattering through a molecular crystal, metaphors can help build up images that lead progressively toward understanding as we probe half-hidden contours of natural phenomena.

Borrowing freely from science, philosophy, and religion, we presume to hope that readers will encounter a newly consilient and satisfying worldview that places humanity, as fractal selves, essentially within the process of the becoming of the universe.

\footnotetext{
"We coined this term in an article published in 1999. See "The Fractal Self and the Organization of Nature: The Daoist Sage and Chaos Theory," in Zygon: Journal of Religion and Science 34, no. 4 (1999): 643-654.
} 
